\title{
AN APPARATUS AND METHOD FOR DETERMINING THE COMPRESSIBILITY OF A GAS AND THE CORRECTION FOR "SUPERCOMPRESSIBILITY"
}

\author{
By Howard S. Bean
}

ABSTRACT

Boyle's law for gases states that the density is directly proportional to the absolute static pressures. All gases depart from this law to some extent, and this is especially true of the industrial fuel gases known as natural gas, for which the departure may be as much as 7 or 8 per cent for a pressure range of 1 to 40 atmospheres. When these gases are metered at pressures of over 5 or 6 atmospheres this departure from Boyle's law becomes important, and a correction must be made to the meter indications by the introduction of a term called the "supercompressibility factor."

In the experimental procedure for determining this factor a sample of gas is collected at high pressure in a steel cylinder of known capacity. Successive small portions of this sample are withdrawn into a burette where their volumes at atmospheric pressure are determined. This is continued until the whole sample has been reduced to atmospheric pressure. The sum of all these volumes at atmospheric pressure is then compared with the volume to which the initial sample of gas would have expanded if it had followed Boyle's law as the pressure was reduced to atmospheric.

The derivation of the equation used in computing the factor from the observational data is given, and the steps to be taken in applying this equation are enumerated. Experience has indicated that the factors thus determined will be correct within \pm 0.002 .

Based on the experience gained with the use of the apparatus described several factors of special interest are discussed. Other methods of determining the supercompressibility factor are also mentioned.

\section{CONTENTS}

I. Introduction

II. Description of the apparatus

1. List of parts. 648

III. Adjustments

IV. Experimental procedure for making a test.

V. Method of computing the supercompressibility factor

1. Constants of the apparatus...

2. Observed quantities.

3. Deduced quantities...

VI. Supplementary notes

1. Volume of tube between valve 2 and cock 3

2. Relation between volumes of cylinder and burette....

3. Precautions to be observed in the use of the constant temperature bath

4. Effects of moisture in connections

5. Comparison of this and other methods of determining the supercompressibility factor. .......................

6. Additional notes on the operation and construction of the apparatus........

\section{INTRODUCTION}

When an orifice or a Venturi tube is employed as a meter for determining the rate of flow of a fluid along a pipe line, the primary quantity to be observed is the pressure drop or differential set up at the meter; but this differential depends on the density of the fluid as well as on the rate of flow, and in order to translate the observed differentials into rates of flow and interpret the readings of the meter, 
the density must be taken into account. With liquids, this is a simple matter; for the density of a liquid is so little affected by any ordinary changes of pressure and temperature that it may, in practice, be treated as a constant and determined, once for all, at any convenient pressure and temperature. But if the fluid in question is a gas, its density varies with the temperature and static pressure in the pipe line, and the interpretation of the readings of the differential becomes more complicated.

It would be advantageous to have a gas densimeter or gravitometer that could be attached directly to the line, or inserted in it and read from outside; but in the absence of such an instrument, it is necessary to observe the temperature and static pressure of the gas, simultaneously with the differential, so that the density in the pipe may be computed from the density measured near atmospheric pressure by means of a specific gravity balance or by some equivalent method.

In many instances, it is sufficiently accurate for commercial orifice meter practice to make the computation by using the ideal gas equation

$$
\frac{P}{\rho T}=R
$$

in which $\rho$ is the density of the gas at the absolute static pressure $P$ and the absolute temperature $T$; and $R$ is the so-called gas constant for the given gas, the numerical value of $R$ depending on the units by which $\rho, P$, and $T$ are measured.

If the density has been found by experiment to be $\rho_{\circ}$ at atmospheric pressure $P_{0}$ and at the temperature $T_{0}$, the density $\rho$ at any other pressure $P$ and temperature $T$ may be found from the equation

$$
\frac{\rho}{\rho_{\circ}}=\frac{P T_{\circ}}{P_{\circ} T}
$$

which follows from equation (1); and if the change of pressure takes place at constant temperature so that $T=T_{\mathrm{o}}$, this reduces to

$$
\frac{\rho}{\rho_{0}}=\frac{P}{P_{0}}
$$

so that the computation of $\rho$ from $\rho_{\mathrm{o}}$ is a very simple matter. Equation (3) is merely a statement of Boyle's law, from which our ordinary ideas on the compressibility of gases are derived.

But in reality, no gas follows equation (1) exactly, and the foregoing: method is not always adequate to the constantly increasing demands for accuracy in commercial measurements. Different gases depart from Boyle's law by different amounts, and both the amount and the direction of the departure depend on the temperature. With most gases, at ordinary temperatures, the density increases with rising: pressure somewhat faster than is indicated by equation (3) or, in other words, the gas is more compressible than if it followed Boyle's law exactly; and this behavior may be represented by writing equation (3) in the modified form

$$
\frac{\rho}{\rho_{\circ}}=y \frac{P}{P_{\circ}}
$$

in which $y$ is a numerical factor slightly greater than 1.00 .1

1 This statement is true when restricted to most natural gases at the temperatures and pressures ordinarily encountered in commercial work. From a general standpoint there are many exceptions to it, one being that for almost all gases the direction of the departure from Boyle's law will be the reverse of that stated, and the value of $y$. will be less than 1.00 at sufficiently high tenperatures or under very high pressures. Another exception is that for some gases, notably hyorogen and helium at ordinary temperature, the value of $y$ is less than 1 . 
This excess of compressibility over what is indicated by Boyle's law may be called the "supercompressibility," and the quantity $y$ will be designated as the "supercompressibility factor."

For air at $60^{\circ} \mathrm{F}$., the value of $y$ at the absolute pressure $P=600$ lbs./in. ${ }^{2}$ is about 1.014 . Hence, even at so high a pressure as this, the computation of the density by means of Boyle's law would involve an error of only 1.4 per cent. And since the orifice meter equations, which need not be discussed here ${ }^{2}$ contain only the square root of the density, the resulting error in the rate of flow deduced from orifice meter readings would be only about 0.7 per cent, an amount which would be negligible in many cases.

With most natural gases, however, the departures from Boyle's law are much greater than with air, and the use of equation (3) without including the correction factor $y$ may result in serious errors, if the gas is metered at high pressure. For example, in the case of a natural gas for which experimental data will be given later (Table 1), the value of the supercompressibility factor, at $32^{\circ} \mathrm{F}$. and 210 lbs./in. ${ }^{2}$ absolute pressure, was found to be nearly 1.04 , and hence the density was nearly 4 per cent greater than that computed from the density at atmospheric pressure by means of Boyle's law. At 600 lbs./in. ${ }^{2}$ the error would probably have been nearly three times as great.

So long as the pressures at which gases were metered were comparatively low, such as $75 \mathrm{lbs}$./in. ${ }^{2}$ absolute, or less, errors of the sort now in question were seldom or never of any commercial importance; but the increasing tendency to raise the pressures on transmission lines has necessarily led to the use of orifice meters at higher pressures, and instances have been reported of measurements at pressures between 700 and 1,000 lbs./in. ${ }^{2}$. This rise in the working pressures, coupled with the increasing demand for more accurate metering, has made supercompressibility a subject of considerable commercial importance.

For several years the Bureau of Standards has been cooperating in researches on orifice meters conducted by the committee on gas measurement of the natural gas department of the American Gas Association. The experiments have been made with natural gas from several fields, and when it was decided to carry on experiments at pipeline pressures of $200 \mathrm{lbs} . / \mathrm{in} .^{2}$ and over, it became necessary to prepare apparatus for determining the supercompressibility factors which would be needed in reducing the observations on the various gases. A description of the apparatus made for this purpose, the mode of operation, and a method of computing the supercompressibility factor from the data obtained are herein presented.

The general outline of the experimental method, which is a classical one, was suggested to the writer as suitable to the purpose in hand by Dr. Edgar Buckingham, who also made some useful suggestions regarding methods of computation. Acknowledgement is likewise due to J. R. Fay for assistance in the preparation of the apparatus.

Before going into details it may be stated that the principle of the method is, briefly, as follows: After a sample of the gas has been collected at high pressure in a steel cylinder of known capacity, successive small portions of the sample are withdrawn into a glass ${ }^{2}$ See Secs. X, XI, and XXVI of B. S. Research Paper No. 49, Discharge Coeficientstof Square-Edged
Orifices for Measuring the Flow of Air.

$101062^{\circ}-30-4$ 
burette where their volumes can be determined at atmospheric pressure. This is continued until the whole sample has been reduced to atmospheric pressure, and the sum of all the volumes at atmospheric pressure is then compared with the volume to which the initial known volume of gas would have expanded if it had been reduced to atmospheric pressure in accordance with Boyle's law.

\section{DESCRIPTION OF THE APPARATUS}

The principal parts of the apparatus and the manner in which they are connected are shown schematically in Figure 1, while Figures $2(a)$ to $(d)$ show the apparatus in detail. The list of parts indicates very briefly the purpose or function of each of the separate parts,

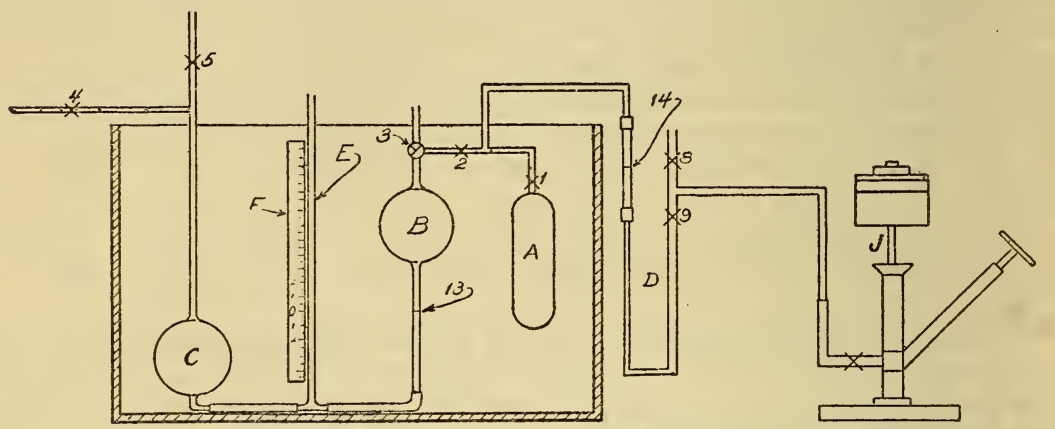

FIGURE 1.-Outiine of the essential features of the apparatus used to determine the supercompressibility factor of a gas

but it is advisable to discuss some of them more fully and to point out certain desirable qualities they should possess.

\section{LIST OF PARTS}

Symbol

Name and description or function

$A$ Gas cylinder for holding gas sample.

$B$ Measuring burette or chamber.

$C$ Mercury reservoir.

$D$ Mercury-oil (or oil) seal.

$E$ Manometer tube.

F Manometer scale.

$G$ Manometer reading slide.

$H$ Agitator tube.

I Trap for spilled mercury.

$J$ Piston gauge.

1 Needle valve, gas cylinder outlet.

2 Needle valve for admitting gas to $B$.

3 3-way glass cock.

$41 / 8$-inch needle valve for admitting pressure to $C$.

$1 / 8$-inch needle valve atmospheric relief for $C$.

Valve for regulating gas (or air) flow to $H$.

Needle valve for filling $A, 1 / 8$-inch.

8 Needle valve for releasing air bubbles from $D$.

9 Needle valve for protecting $J$.

10 Needle valve (all steel) used in filling $D$ with mercury.

11 Extension for operating valve 1.

12 Extension for operating cock 3.

13 Etched line about lower neck of $B$.

14 Etched line about middle of glass tube portion of $D$. *

15 Extension rod for moving $G$.

16 Guide rod on which $G$ slides.

17 Windows in box sides. 
The cylinder $A$ (fig. 1) need not be very large-the capacity of the one used was about 36 cubic inches. It should be strong enough to withstand any pressure at which a test is to be started, without expanding appreciably. While not essential, it is convenient to have the cylinder provided with two valves to facilitate purging and filling. The capacity of the cylinder, with valve 7 (fig. $2(a)$ ) closed, to the outlet connection of valve 1 must be determined to an accuracy of about 1 part in 2,000.

The burette, $B$, should be of strong and fairly heavy glass. In the upper stem is the 3 -way cock, 3 , by means of which the burette may be opened to the atmosphere for purging, or to the tube for connecting it with $A$. About the lower neck, a short distance below the body of the burette, is an etched line, 13. The capacity of the burette, between the 3-way cock as the upper limit and the etched line as the lower limit, must be determined to an accuracy of 1 in 2,000 or better. (The passages in the plug of the 3 -way cock are not included in this volume.)

In order to determine the pressures with a satisfactory degree of accuracy, it is necessary to use a dead-weight piston gauge $J$. (Fig. 1.) This, of course, will require that there be at hand the necessary small weights for reading pressures to $0.1 \mathrm{lbs} . / \mathrm{in} .{ }^{2}$. The use of the piston gauge makes it necessary to have the trap $D$ between it and the gas cylinder $A$. This trap is essentially a special manometer about 15 inches high, most of it being built up of suitable small pipe fittings, but the upper 5 or 6 inches of the leg to which $A$ is attached consists of a heavy gauge glass tube, thoroughly annealed and with each end set into a special pipe coupling with Khotinsky cement. Around the center of the glass tube is an etched line (14). The upper end of the other leg of the trap is provided with two valves, one on either side of the tee to which $J$ is connected. The length of this leg is such that the offiset of the tee is slightly below the level of the etched line (14).

Small bore (1/8 inch outside diameter) copper tubing is used to connect the trap with $A$, and enough bends are made in the trap end to guard against putting an excessive strain on the glass. There is a tee in the tube near the cylinder end, and the needle valve 2 is connected to one branch of this tee. The outlet of this valve is connected to the burette $B$ by as short a tube as possible. The capacity of the tubing between valves 1 and 2 and the etched line 14 must be determined to the same absolute accuracy as the other volumes.

The manometer tube $E$ is the elongated offset of a tee. The run of the tee forms a part of the line which joins the lower neck of $B$ with the reservoir $C$. It is desirable to have the tube $E$ long enough to extend even with or above the top of the atmospheric outlet from the 3 -way cock 3 .

The scale $F$, which is attached to or fastened beside the tube $E$, should be about 10 inches long with the zero near the center and the graduations numbered both ways. In the apparatus now being described, this scale is of glass with etched graduations and figures, and the smallest division of the scale is $1 / 20$-inch.

The reservoir $C$ should have a capacity about one-tenth larger than that of $B$. The relative elevations of $B$ and $C$ should be such that the etched line 13, on the lower neck of $B$ is level with or higher than the opening in to the upper neck of $C$. This upper neck of $C$ is connected to the valves 4 and 5 . Through 4 gas or air under pressure may be admitted to $C$ for forcing the mercury over into $B$ while 5 is a relief valve. 


\section{ADJUSTMENTS}

After the apparatus has been assembled it is necessary to make a few adjustments before it is ready for use. The first is to adjust the quantity of sealing fluid in the trap $D$. This is very easy if only oil is to be used in the trap, as it is only necessary to pump in oil from the piston gauge until it stands about one-half inch above the etched line 14. Air bubbles may be released through valve 8 . When mercury is used with oil, this adjustment is not so easy. Mercury is introduced into the trap through the all-steel needle valve 10

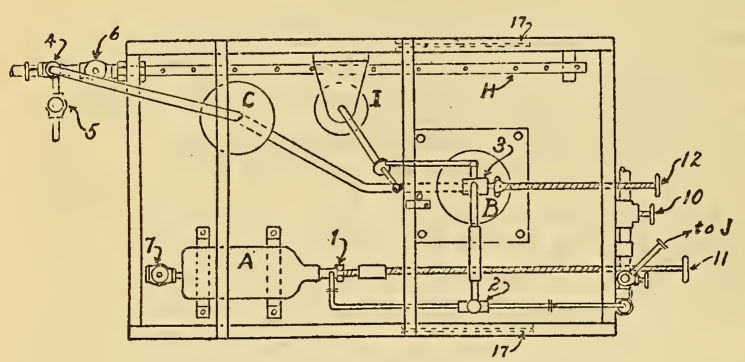

a

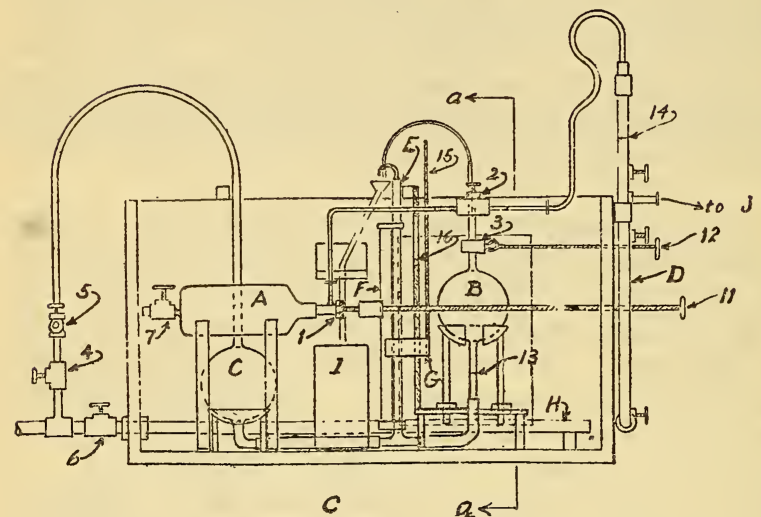

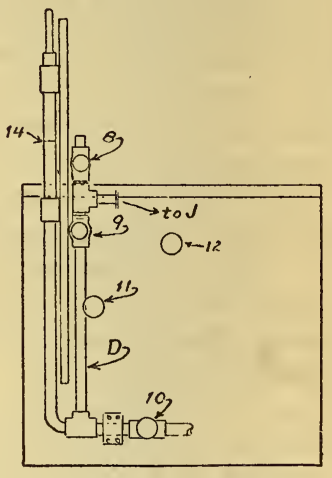

6

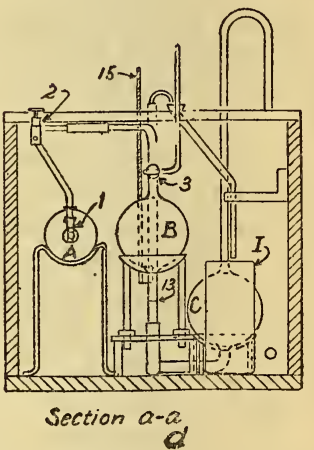

Figure 2.-Apparatus used to determine the supercompressibility factor of a gas

$a$, Plan view; $b$, elevation of right end showing assembly of the mercury-oil seal; $c$, front elevation with side of tank removed; $d$, elevation of section $a-a$.

(Fig. $2(a)$.) Enough mercury is used to fill the gauge glass side to one-half inch above the etched line 14, and to extend about 3 inches up the other leg. The rest of the trap and the tube to the piston gauge is filled with oil. The procedure used to do this is to fill both legs of the trap with mercury to a height of about 3 inches. Then after filling the piston gauge side with oil, valve 9 is closed and the quantity of mercury needed to complete the filling of the gauge glass side is introduced through valve 10. It is necessary to get out all the air bubbles from the oil in the trap and tubing to the piston gauge.

The use of mercury in one leg of the trap introduces an unbalanced pressure on the piston gauge. It would be possible to have this 
column of mercury just balance the weight of the piston; but in the particular apparatus being described a steel disk had been attached to the plunger as a part of an arrangement to keep the plunger from being blown from the oil cylinder, and to balance this added weight on the plunger would have required an undesirably long trap. In such a case as the present one it is necessary to calibrate the combined mercury-oil-piston gauge system, and this is done by applying air or gas under pressure to the mercury side of the trap until the plunger, with all weights removed, begins to rise and the surface of the mercury to fall. When the top of the mercury is in the plane of the etched line 14 the amount of pressure being applied is read on a mercury manometer temporarily connected ahead of the trap. On account of the effects of friction and viscosity upon the plunger, it is best to have the pressure increasing or decreasing very slowly and to read the auxiliary manometer quickly as the surface of the mercury in the trap creeps past the etched line. The value used is then the average obtained with falling and rising pressures.

The next adjustment to be made is that of the position of the scale $F$, beside the manometer tube $E$. To do this the cock 3 and the valve 5 are opened so that both $B$ and $C$ as well as $E$ are open to the atmosphere. Mercury is then poured into the system until $C$ is full and

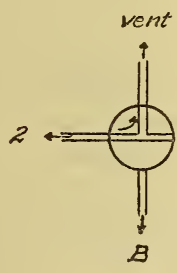

(a)

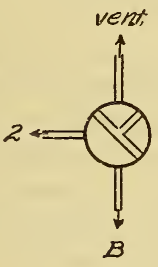

(b)

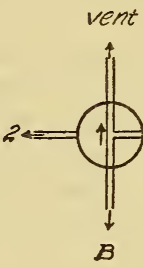

(c)

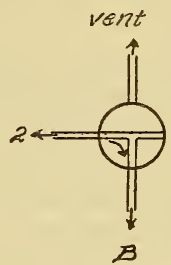

(d)

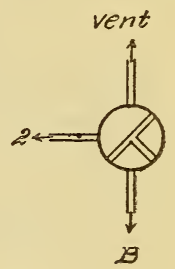

(e)

FIgURE 3.-Successive positions of the core of the 3-way cock, 3

the surface of the mercury in the lower neck of $B$ is exactly in the plane of the etched line 13. The scale is then moved up or down until its zero is exactly level with the surface of the mercury in $E$, and is finally fastened in that position. After completing this adjustment, enough mercury is removed from the system so that the surface of the mercury in the lower neck of $B$ will be one-fourth to three-eighths inch below the etched line 13. This is done so that the mercury may not foul the inner surface of the lower neck of $B$ right at the etched line, by standing there when the apparatus is not in use.

\section{EXPERIMENTAL PROCEDURE FOR MAKING A TEST}

When the foregoing adjustments have been made, the apparatus is ready for use. The cylinder $A$ is charged with the gas to be tested, put in place, and connected to the tube from $D$ and 2 . The tank in which the apparatus is mounted is filled with water and the water stirred to insure a uniform temperature. To purge the tubing, cock 3 is turned so as to open the line from valve 2 to the atmosphere, as shown by position (a) of Figure 3 . With valves 2 and 9 closed, valve 1 is cracked so that the pressure within the tubing becomes equal or nearly equal to that in $A$. After closing valve 1 , 
valve 2 is opened until the excess pressure in the tubing is dissipated and then closed again, and cock 3 is closed by turning it to position (b) of Figure 3. A second purging is not usually necessary. Valve 1 is again cracked so that the pressure within the tubing will slowly build up to the full pressure within $A$, after which valve 1 is opened a full turn or more. Weights are now placed upon the piston gauge, and as valve 9 is opened these are adjusted so that with the plunger floating freely, the top of the fluid in the trap is in the plane of the etched line 14. The weights on the piston gauge are noted and recorded Next, the cock 3 is turned so as to open $B$ to the air while closing the tube to valve 2 (position (c) fig. 3) and, with valve 5 closed, valve 4 is cracked so as to admit air or gas under a low pressure (10 to $20 \mathrm{lbs}$./in. ${ }^{2}$ ) into $C$, thus forcing mercury from $C$ into $B$. Just as the mercury in $B$ reaches the edge of the 3 -way cock, valve 4 is closed and cock 3 is turned so as to connect $B$ to the line from valve 2 . (Position (d) fig. 3.) As valve 5 is opened slowly, valve 2 is cracked thus letting gas from $A$ into $B$ as the mercury flows back into $C$. As the surface of the mercury in $B$ enters the lower neck, valve 2 is nearly closed, and it is closed tight when the surface of the mercury has fallen just below the etched line 13. Cock 3 is next turned so as to close all entrances. (Position (e) fig. 3.) By closing valve 5 and cracking valve 4 , the surface of the mercury is brought back just to the plane of the etched line, 13, and the pressure of the gas in $B$ is then obtained by reading on $F$ the position of the mercury in $E$.

This completes one cycle of operations and we proceed with another by readjusting the weights on the piston gauge to balance the new pressure in $A . \quad B$ is again filled with mercury and the operation continued, as just described. This cycle of operations is repeated until the gauge pressure of the gas in $A$ has been reduced to zero or nearly zero. After the last filling of $B$ with gas, valve 2 and cock 3 are left open so that $A$ and $B$ are in free communication. The surface of the mercury in the trap $D$ is brought to the plane of the etched line 14 by manipulation of the oil pump on the piston gauge. The pressure in $B$ is now determined as before and this is also the pressure of the gas finally remaining in $A$.

This final gauge pressure must be low enough to be measured on the short manometer $E$ which, in the present apparatus, had a range of about \pm 5 inches of mercury, or somewhat less than \pm 2.5 lbs./in. ${ }^{2}$, and to insure this result it may be necessary to adjust the length of the last few steps in the process. When the gauge pressure in $A$ has fallen to about $100 \mathrm{lbs}$./in. ${ }^{2}$ it is divided by the average fall of pressure during the three or four immediately preceding cycles. If the quotient is very nearly a whole number, it is evident that this whole number of additional cycles, similar to those immediately preceding, will reduce the gauge pressure so nearly to zero that it will be within the range of the manometer $E$. In this case no change from the previous rate of withdrawal of gas from $A$ is required. Otherwise, the rate of withdrawal for the remaining cycles must be increased or decreased sufficiently that a whole number of cycles will leave the final residual pressure within the range of the manometer $E$.

Whether such an adjustment may be needed and, if so, where it should be started, depends on the relative volumes of $A$ and $B$ and on the range of $E$, so that no general numerical directions are possible, but the practical working of the process is illustrated by the figures in the third and fourth columns of Table 1. 


\section{METHOD OF COMPUTING THE SUPERCOMPRESSIBILITY FACTOR}

In developing the equations needed for the computations, the following notation and definitions will be employed.

\section{CONSTANTS OF THE APPARATUS}

$V_{a}=$ the volume of the cylinder $A$ and the adjacent tubing, out to the valve 2 and the etched mark 14 .

$V_{b}=$ the volume of the burette, from the mark 13 to the plug of cock 3 when closed.

Only the ratio of these volumes appears in the final equation, so that their values may be expressed in terms of $K\left(\right.$ lbs. $/$ in. $\left.{ }^{2}\right)=$ the trap constant.

It is the gas pressure that must be applied at the top of the trap in order to force the mercury surface down to the mark 14 when the piston of the gauge is floating freely with all the weights removed. It is measured, once for all, on an auxiliary mercury column, as described above under the heading "Adjustments," and then expressed in lbs./in. ${ }^{2}$.

\section{OBSERVED QUANTITIES}

$W\left(\right.$ lbs./in. $\left.{ }^{2}\right)=$ the pressure due to the weights on the piston gauge.

It is the total added weight in pounds divided by the cross section of the piston in square inches. For convenience, each of the movable weights should be marked with its equivalent in terms of pressure, so that the value of $W$ may be read directly.

$B$ (inches $\mathrm{Hg}$ ) $=$ the barometer reading, corrected to $32^{\circ} \mathrm{F}$.

$p_{b}$ (inches $\mathrm{Hg}$ ) $=$ the reading of the manometer $E$.

For the highest possible accuracy, $p_{b}$ should also be corrected to $32^{\circ} \mathrm{F}$., but in most cases that would be a useless refinement.

$t\left({ }^{\circ} \mathrm{F}.\right)=$ the temperature of the water bath.

It is assumed that $t$ remains sensibly constant throughout the complete set of measurements on any one sample of gas collected in $A$. Before starting the observations, time must be allowed for the newly introduced sample to come to the temperature of the bath.

$n=$ the number of cycles of operations in the whole set of measurements on the sample.

\section{DEDUCED QUANTITIES}

$B_{s}$ (lbs./in. $\left.{ }^{2}\right)=$ the barometric pressure expressed in pounds per square inch.

The reduction is made by means of the relation

$1\left(\right.$ inch $\mathrm{Hg} 32^{\circ}$ F.) $=0.4912\left(\mathrm{lbs} . /\right.$ in. $\left.^{2}\right)$

$P_{a}\left(\mathrm{lbs} . /\right.$ in. $\left.^{2}\right)=$ the absolute pressure in the cylinder $A$ and the adjoining tubing as far as the valve 2 and the mark 14 , when the valve 1 is open.

$$
P_{a}=W+K+B_{s}
$$


$P_{b}$ (lbs./in. $\left.{ }^{2}\right)=$ the absolute pressure in the burette.

$$
P_{b}=0.4912\left(B+p_{b}\right)
$$

$V_{s}=$ the volume that would be occupied by the mass of gas which fills the cylinder and tubing at the pressure $P_{a}$, if it were allowed to expand to atmospheric pressure.

$y=$ the supercompressibility factor.

$y$ is defined as the ratio of the actual density at $P_{a}$ to the "theoretical" density computed from the density at atmospheric pressure, on the assumption of Boyle's law.

If $m$ denotes the initial mass of gas contained in the volume $V_{a}$ at the pressure $P_{a}$, we have the relation

$$
\text { actual density at } P_{a}=\frac{m}{V_{a}}
$$

When expanded to the volume $V_{s}$ which it occupies at the pressure $B_{s}$, the gas has the density $m / V_{s}$; and if it were then recompressed to $P_{a}$, its density computed from Boyle's law would be

$$
\text { theoretical density at } P_{a}=\frac{m}{V_{s}} \times \frac{P_{a}}{B_{s}}
$$

Hence we have, from the definition of $y$,

$$
y=\frac{m}{V_{a}} \div\left(\frac{m}{V_{s}} \times \frac{P_{a}}{B_{s}}\right)=\frac{V_{s} B_{s}}{V_{a} P_{a}}
$$

in which $V_{a}, P_{a}$, and $B_{s}$ are known, and it remains to find the value of $V_{s}$.

Let subscript $k$ be used to specify quantities referring to the $k$ th cycle of operations: $k$ may have any value from 1 to $n$, inclusive.

At the start of the $k$ th cycle the pressure in the cylinder and tubing is $\left(P_{a}\right)_{k}$. The gas withdrawn into the burette fills the volume $V_{b}$ at the absolute pressure $\left(P_{b}\right)_{k}$; and if its pressure were changed to $B_{s}$, its volume would be

$$
v_{k}=V_{b} \frac{\left(P_{b}\right)_{k}}{B_{s}}
$$

for $P_{b}$ is always so nearly equal to the outside barometric pressure $B_{s}$ that the departure from Boyle's law over this small range of pressure is entirely negligible.

Starting with the $k$ th cycle and proceeding through the $n$th and last, the combined volume, at the pressure $B_{s}$, of all the portions of gas withdrawn into the burette is

$$
\begin{aligned}
v_{k}+v_{k+1}+---+v_{n} & =\frac{V_{b}}{B_{s}}\left[\left(P_{b}\right)_{k}+\left(P_{b}\right)_{k+1}+---+\left(P_{b}\right)_{n}\right] \\
& =\frac{V_{b}}{B_{s}} \sum_{n}^{k} P_{b}
\end{aligned}
$$


At the end of the $n$th cycle, the cylinder and tubing remain filled with gas at the final pressure $\left(P_{b}\right)_{n}$, and if this residual gas were also brought to the pressure $B_{s}$, its volume would be

$$
v_{r}=V_{a} \frac{\left(P_{b}\right)_{n}}{B_{s}}
$$

All the gas is now accounted for and we have

$$
\left(V_{s}\right)_{k}=v_{k}+v_{k+1}+--+v_{n}+v_{r}
$$

or by equations (12) and (13)

$$
\left(V_{s}\right)_{k}=\frac{1}{B_{s}}\left[V_{s} \sum_{n}^{k} P_{b}+V_{a}\left(P_{b}\right)_{n}\right]
$$

and upon substituting this value in equation (10) we have

$$
y_{k}=\frac{1}{\left(P_{a}\right)_{k}}\left[\frac{V_{b}}{V_{a}} \sum_{n}^{k} P_{b}+\left(P_{b}\right)_{n}\right]
$$

as our final equation for computing the value of the supercompressibility factor at the pressure $\left(P_{a}\right)_{k}$.

As already remarked, the volumes $V_{a}$ and $V_{b}$ appear only as a ratio, and it may be noted that the pressures also occur in both numerator and denominator of equation (16). Hence, they might equally well have been expressed in inches of mercury instead of pounds per square inch, the only requirement being that $P_{a}$ and the $P_{b}$ 's shall be expressed in terms of the same unit.

The trap constant $K$ and the volume ratio $\left(V_{b} / V_{a}\right)$ having been determined once for all, the computation of $y$ from the observed data by means of equation (16) may conveniently be carried out in the following steps.

1. For each cycle of operations, compute the values of $P_{a}$ and $P_{b}$ (equations (5), (6), and (7)).

2. Starting with the $n$th or last value of $P_{b}$, add to it the $(n-1)$ th value of $P_{b}$; add to this sum the $(n-2)$ th value, and so on, thus finding the successive values of $\sum_{n}^{k} P_{b}$ for all the cycles, from the last to the first.

3. Multiply each of these sums by the volume ratio $\left(V_{b} / V_{a}\right)$ and to this product add the $n$th value of $P_{b}$.

4. Divide each of the quantities thus obtained by the corresponding value of $P_{a}$. The quotient is the required value of $y$.

A sample set of observed and computed values is given in Table 1. If the values of $y$ are plotted against those of $P_{a}$, and if a mean curve is drawn through the resulting band of points, it appears that values of $y$ read from the curve will nowhere be in error by more than 0.002 , if the volume ratio was accurately determined. The value of $\sqrt{y}$ needed in orifice meter computations will therefore be correct within 1 part in 1,000 and we may conclude that experiments of the sort from which the data in Table 1 were obtained are amply precise for ordinary commercial purposes. 


\section{TABLE 1.-Compressibility test data}

Sample collected at Daly station.

Date of collection, August 1, 1928.

Pressure when collected, 210-pound gauge.

Barometer:

At start, 29.42 at $72^{\circ} \mathrm{F}$.

At end, 29.44 at $73^{\circ} \mathrm{F}$.

Barometer corrected to $32^{\circ} \mathrm{F}$.

$B=29.31$ inches.

$B_{8}=14.40 \mathrm{lbs}$./in. ${ }^{2}$
Test No. XX.

Date of test, August 1, 1928.

Test made by, H. S. B.

\section{Room temperature:}

$73^{\circ} \mathrm{F}$.

Trap constant, $K=+9.66 \mathrm{lbs} . / \mathrm{in} .^{2}$

Volume ratio, $V_{b} / V_{a}=0.8672$.

\begin{tabular}{|c|c|c|c|c|c|c|c|c|c|}
\hline \multicolumn{4}{|c|}{ Observed values } & \multicolumn{6}{|c|}{ Computed values } \\
\hline$\stackrel{k}{\text { (cycle No.) }}$ & $\begin{array}{l}\text { Bath tem- } \\
\text { perature }\end{array}$ & $W$ & $p_{b}$ & $P_{b}$ & $\underset{n}{\stackrel{k}{S} P_{b}}$ & $\frac{V_{\mathrm{b}}}{V_{\mathrm{a}}} \sum_{n}^{k} P_{\mathrm{b}}$ & $\begin{array}{l}\frac{V_{\mathrm{b}}}{V_{\mathrm{a}}} \sum_{n}^{k} P_{b} \\
+\left(P_{b}\right)\end{array}$ & $P_{a}$ & $y$ \\
\hline & ${ }^{\circ} F$. & $\begin{array}{r}\text { Lbs./in. }{ }^{2} \\
186.2 \\
174.6 \\
162.8 \\
151.2\end{array}$ & $\begin{array}{r}\text { Inches, } \mathrm{Hg} \\
-0.56 \\
+.67 \\
-.32 \\
-.52\end{array}$ & $\begin{array}{r}\text { Lbs./in. }{ }^{2} \\
14.12 \\
14.73 \\
14.24 \\
14.14\end{array}$ & $\begin{array}{l}233.04 \\
218.92 \\
204.19 \\
189.95\end{array}$ & $\begin{array}{l}202.09 \\
189.85 \\
177.07 \\
164.72\end{array}$ & $\begin{array}{l}218.26 \\
206.02 \\
193.24 \\
180.89\end{array}$ & $\begin{array}{r}L \text { Ls./in. } .^{2} \\
210.26 \\
198.66 \\
186.86 \\
175.26\end{array}$ & $\begin{array}{l}1.038 \\
1.037 \\
1.034 \\
1.032\end{array}$ \\
\hline & 32.5 & $\begin{array}{l}139.8 \\
128.2 \\
116.5 \\
104.7\end{array}$ & $\begin{array}{l}-.41 \\
-.26 \\
-.30 \\
-.26\end{array}$ & $\begin{array}{l}14.20 \\
14.27 \\
14.25 \\
14.27\end{array}$ & $\begin{array}{l}175.81 \\
161.61 \\
147.34 \\
133.09\end{array}$ & $\begin{array}{l}152.46 \\
140.15 \\
127.77 \\
115.42\end{array}$ & $\begin{array}{l}168.63 \\
156.32 \\
143.94 \\
131.59\end{array}$ & $\begin{array}{l}163.86 \\
152.26 \\
140.56 \\
128.76\end{array}$ & $\begin{array}{l}1.029 \\
1.027 \\
1.024 \\
1.022\end{array}$ \\
\hline $\begin{array}{l}9-- \\
10- \\
11 \\
12-\end{array}$ & 32.6 & $\begin{array}{l}93.0 \\
80.7 \\
69.0 \\
56.9\end{array}$ & $\begin{array}{l}-.05 \\
-.32 \\
-.22 \\
+.94\end{array}$ & $\begin{array}{l}14.37 \\
14.24 \\
14.29 \\
14.86\end{array}$ & $\begin{array}{r}118.82 \\
104.45 \\
90.21 \\
75.92\end{array}$ & $\begin{array}{r}103.04 \\
90.58 \\
78.23 \\
65.84\end{array}$ & $\begin{array}{r}119.21 \\
106.75 \\
94.40 \\
82.01\end{array}$ & $\begin{array}{r}117.06 \\
104.76 \\
93.06 \\
80.96\end{array}$ & $\begin{array}{l}1.018 \\
1.019 \\
1.014 \\
1.013\end{array}$ \\
\hline $\begin{array}{l}13 \\
14- \\
15 \\
16\end{array}$ & 32.9 & $\begin{array}{r}44.4 \\
31.7 \\
18.9 \\
6.1\end{array}$ & $\begin{array}{l}+1.16 \\
+1.10 \\
+1.18 \\
+3.61\end{array}$ & $\begin{array}{l}14.97 \\
14.94 \\
14.98 \\
16.17\end{array}$ & $\begin{array}{l}61.06 \\
46.09 \\
31.15 \\
16.17\end{array}$ & $\begin{array}{l}52.95 \\
39.97 \\
27.01 \\
14.02\end{array}$ & $\begin{array}{l}69.12 \\
56.14 \\
43.18 \\
30.19\end{array}$ & $\begin{array}{l}68.46 \\
55.76 \\
42.96 \\
30.16\end{array}$ & $\begin{array}{l}1.010 \\
1.007 \\
1.005 \\
1.001\end{array}$ \\
\hline
\end{tabular}

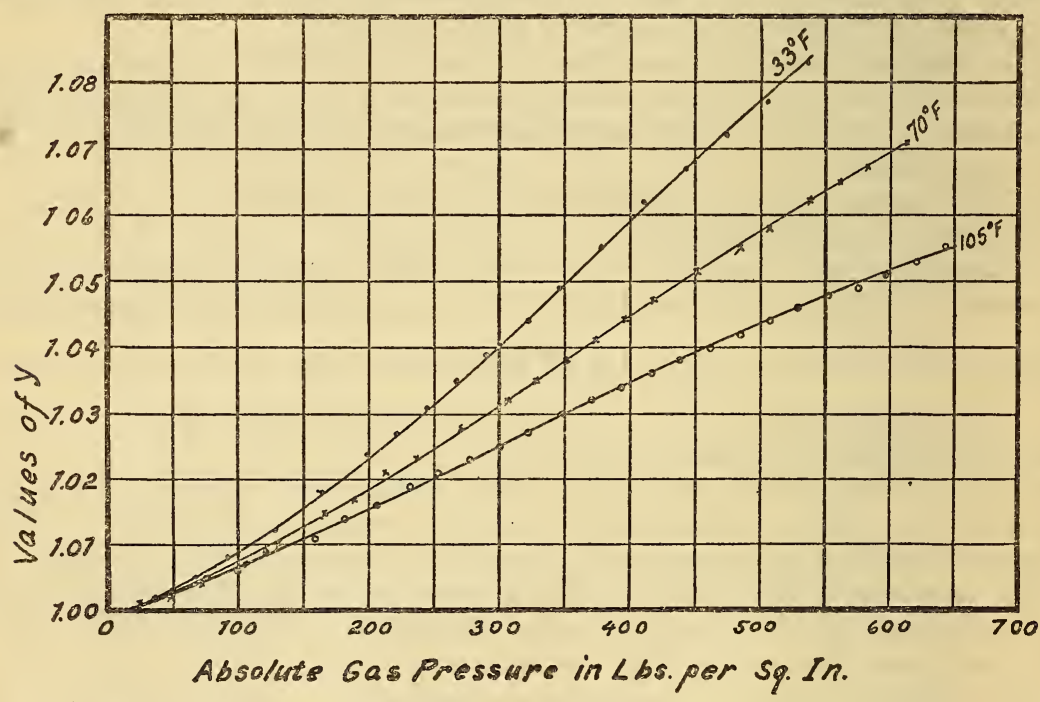

FIGURE 4.-The effects of temperature upon the supercompressibility correction factor

A large gas cylinder was filled with gas to a pressure of about 1,000 pounds, and samples of gas were drawn from this cylinder when filling the sample cylinder, $A$. Analysis of this gas showed it was 98.7 per cent methane. 


\section{SUPPLEMENTARY NOTES}

\section{VOLUME OF TUBE BETWEEN VALVE 2 AND COCK 3}

It may have been noted that the small volume of the tubing between valve 2 and cock 3 which, for convenience, we will designate by $u$, has been omitted from all calculations. It will now be shown that this omission has introduced no appreciable error. Before the burette is filled with gas the first time, the tubing has been purged and the section $u$ remains full of gas at sensibly atmospheric pressure. Whenever the burette is being purged of gas, $u$ will always remain full of gas, regardless of whether cock 3 is turned so as to entrap the gas therein or not. (It is better practice to turn 3 so as to close off the tube from valve 2 ; then even if 2 should leak a little the gas will not escape unmeasured.)

Now, the actual quantity or mass of gas entrapped within $u$ during each purging of $B$ will depend upon the pressure in $B$ at the time cock 3 is turned to close off $u$. But this pressure will usually be very nearly atmospheric if the experimental work is done with reasonable care. Hence, the successive masses of gas within $u$ will be sensibly equal, and if they were exactly equal it would be unnecessary to consider them at all. This applies to all fillings of $B$ from 1 to $(n-1)$, inclusive.

After the last or $n$th filling of $B$, all valves between $A$ and $B$ are left open. If at this time it should happen that $P_{b}$ were just equal to $B_{s}$, this last mass of gas in $u$ would be equal to the mass of gas in $u$ just prior to the first filling of $B$. Obviously, in this case no correction would be needed.

In most cases $P_{b}$ will be either greater or less than $B_{s}$ and the last mass of gas in $u$ will be correspondingly greater or less than the first. In these cases it is true that, in principle, a correction should be applied, and the amount of this correction could readily be determined. But it is also evident that since this correction would be applied to only the last of the $n$ masses of gas taken from $A$ into $B$, the correction will have no sensible influence upon the results, if the volume of $u$ is small compared to that of $B$. (In the apparatus shown in fig. 2 the ratio of $u$ to $V_{b}$ is less than 1:500.)

\section{RELATION BETWEEN VOLUMES OF CYLINDER AND BURETTE}

If the volumes of the cylinder and burette are made approximately equal, each filling of the burette will reduce the pressure in the cylinder by approximately 1 atmosphere, a conveniently simple relation. This, however, might require an undesirably large number of steps or cycles, and when the initial cylinder pressure is 300 pounds or over, it is desirable to have fewer steps than a 1 to 1 ratio of volumes would require. In such cases it would be convenient to have the cylinder and burette volumes in the ratio of 1 to 2 or 1 to 3 . Such a relation of volumes would not lessen the accuracy and usefulness of the results, but would greatly shorten the time required to make the test. In a commercial apparatus it might be convenient to have cylinders of two or more sizes, which would permit the use of the size most suitable to the pressure at which the sample was collected. 


\section{PRECAUTIONS TO BE OBSERVED IN THE USE OF THE CONSTANT TEMPERATURE BATH}

With the present apparatus as shown in Figure 2, a water bath is used to maintain the gas and all parts of the apparatus, except the trap and piston gauge, at a constant temperature. This makes it necessary to be very careful that no water enters any of the tubing or joints while operating the apparatus or when removing and replacing: the cylinder. If water should get into the burette or tubing, it must be removed. In the case of water in the burette, it may be possible to remove most of it by removing the plug of the cock 3 and forcing the water out with the mercury. The final traces of moisture may best be removed by repeatedly filling the burette with warm air that has been passed through a dryer.

One reason for maintaining the temperature constant is that the magnitude of the supercompressibility factor depends upon the temperature of the gas at which the test is made. In general, the lower the temperature the higher will be the value of this factor, as is illustrated by Figure 4; but over a limited temperature range, for instance, from $50^{\circ}$ to $70^{\circ} \mathrm{F}$., the change in the value of this factor will probably be small for most industrial gases. The effects of such small variations in the factor upon the measurements of gas will probably be negligible from the standpoint of commercial metering, particularly in measurements by orifice meters where the value of $y$ occurs in the computations only as the square root.

A more important reason for using a water bath is that computations by means of the equations given above are all based on the assumption that the temperature of the gas remains constant throughout each complete set of observations. If this condition is not satisfied, the equations may be modified to take account of the changes of temperature; but the computations are then more complicated and it is better to keep the temperature as nearly constant as practicable. A variation of the order of $1^{\circ} \mathrm{F}$., such as is shown in Table 1 , may be disregarded.

\section{EFFECTS OF MOISTURE IN CONNECTIONS}

In the preceding section attention is called to the necessity of keeping the burette and tubing dry and free from all water and oil particles. Since failure to do this may lead to very misleading results, it will be well to consider this subject in more detail.

At the start of a test the sample cylinder is filled with gas under a relatively high pressure. It may be saturated with water and oil vapors at that pressure and the bath temperature. As the gas is drawn out by successive fillings of the burette the gas pressure decreases and the gas in both burette and cylinder becomes relatively drier. If then this gas comes in contact with particles of water, some of this water will be picked up in the form of vapor. This acquired vapor will increase the total pressure of the gas in the burette over that which the gas alone would have, with the result that the value of $y$ will be increased. 
If the water particles are in the burette or the connections between the burette and valve 2 , the absorption of moisture may take place nearly uniformly throughout the test. The probable effect in this case would be to increase the slope of the $y-P_{a}$ curve more or less uniformly. However, this condition is not the most likely to occur. Firstly, it is seldom necessary to make any changes in the connections of this part of the system once it is put together. Secondly, the most likely place for water to enter would be about the plug of the 3-way cock and even if this should happen the presence of the water would be noticeable.

In the particular apparatus here described the most probable place for water particles to lodge is in the tubing between valves 1 and 2 , because the union joint near valve 1 was broken and made each time the cylinder was filled. During a test the gas pressure within this part of the tubing is the same as in the cylinder. If there is any

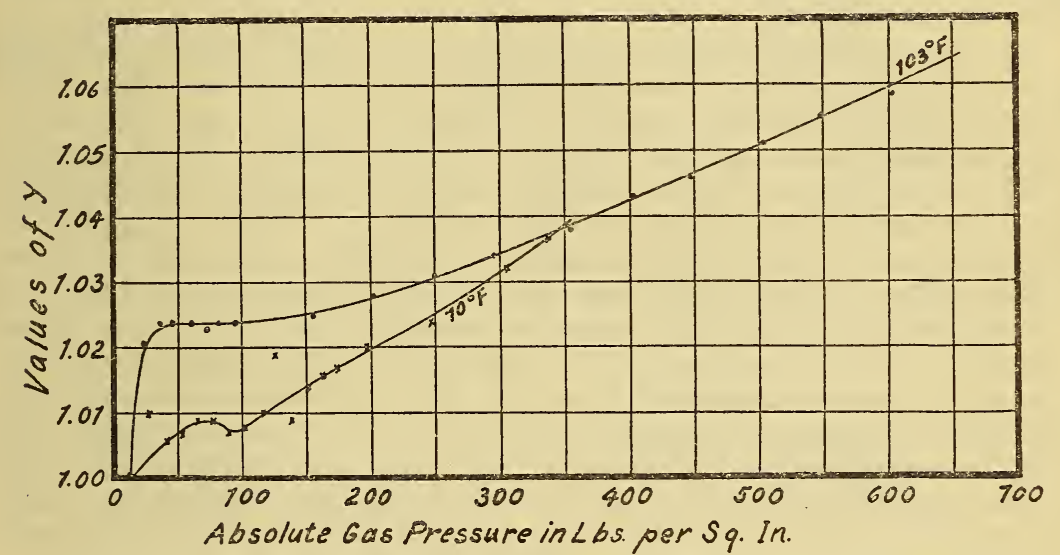

FIGURE 5.-The effects of water in the connections upon the supercompressibility correction factor

The samples of gas used in these tests were from the same source as those from which the curves in Figure 4 were obtained

water within this tubing, the amount that will be absorbed by a gram of gas will be extremely small at the higher pressures, but will increase rapidly as the gas pressure approaches atmospheric. This will cause the increments in $y$ to be excessively large at the pressures close to atmospheric, as illustrated in the curves of Figure $5 .^{3}$

\section{COMPARISON OF THIS AND OTHER METHODS OF DETERMINING THE SUPERCOMPRESSIBILITY FACTOR}

In the method just described the sample of gas which is used represents the actual conditions of pressure and degree of saturation with water and oil vapors that existed in the line at the time the sample was collected. Also, by regulation of the bath water temperature the test can be made at the same temperature as that of the gas in the line. Since this temperature is maintained constant, while the pressure is steadily decreased, no condensation of any vapors mixed with the gas will take place. Furthermore, if the test is carefullly

${ }^{3}$ After the particular tests were completed, from which the curves in fig. 5 were obtained, small drops of water were found in the tubing near valve 1 . 
made, there will be no absorption of additional vapors by the gas, with the result that the $y-P_{a}$ curve obtained from the test represents the magnitude of the deviation from Boyle's law within the degree of precision mentioned in a preceding section.

In some instances which have been reported, a sample of the gas is collected in a large cylinder at the line pressure. The volume at atmospheric pressure is then obtained by metering the gas through a small laboratory wet gas meter, or by the displacement of water in an inverted bell. This method has been used in a few instances. However, in at least one group of tests made by this method, of which a report has been published, ${ }^{4}$ the curves obtained are similar to those of Figure 5. While this similarity suggests that the sharp rise in the $y-P_{a}$ curve at low values of $P_{a}$ was due to the presence of water in some part of the apparatus with which those tests were made, it would be impossible to assign that as the real cause without knowing all of the conditions.

Another method consists of starting with a small sample of the gas at atmospheric pressure and compressing it over mercury. The several pressures applied and the corresponding volumes are read by suitable instruments, and from the data thus obtaineu the $y-P_{a}$ curve can be determined. Under suitable conditions a very high degree of accuracy may be obtained by this method, and it is well suited to laboratory work on pure, dry gases. However, this method is not suitable when an industrial gas, and particularly a natural gas, is to be studied, because these gases are always more or less saturated with water and oil vapors, particularly when at atmospheric pressure. As such a gas is compressed a part of these vapors is condensed with the result that the deviation from Boyle's law will be exaggerated, and this exaggeration will be relatively more at the lower pressures. In the report ${ }^{5}$ on one group of tests made by this method the curves indicate that some condensation occurred as the gas was compressed, though possibly not enough to be readily observed.

There is also an analytical method which has been tried and possibly used in a few instances. With this method it is assumed that the supercompressibility factors for a gas mixture may be calculated on the basis of a chemical analysis of the mixture if we know the supercompressibility factors for all of the pure constituents. ${ }^{6}$ A chemical analysis of a gas sufficiently accurate for this purpose can usually be obtained fairly easily as most companies interested in this question have both the men and the equipment for making such tests. The supercompressibility factors for the pure gases may be obtained from tables in various reference books or may be calculated by one of the several equations of state. ${ }^{7}$ In a few instances in which comparisons have been made it was found that results given by this analytical method usually agreed fairly well with the results obtained by the method described in this paper. However, the use of this method is limited because there are few or no reliable data on several of the common constituents of natural gases.

4 Corrections to Boyle's Law for High-Pressure Gas, by D. E. Silcox, Mech. Eng., p. 897; November, 1925.

${ }_{3}$ Deviation of Natural Gas from Boyle's Law, by R. F. Earhart and S. S. Wyer, Trans. A. S. M. E., 38, p. 285; 1916.

6 The Compressibility of Natural Gas at High Pressures, by G. A. Burrell and I. W. Robertson, Bureau of Mines Tech. Paper No. 131.

7 Relations Between Temperature Pressure and Density of Gases, Circular of the Bureau of Standards, No. 279 . 


\section{ADDITIONAL NOTES ON THE OPERATION AND CONSTRUCTION OF THE APPARATUS}

Since the apparatus as described uses mercury, it is necessary that there be no parts or fittings, particularly in the mercury and gas leads, that the mercury will attack. This rules out brass fittings and soldered joints for most parts, although pure copper tubing may be used with but little danger of trouble. However, when the water bath is used, it is desirable to avoid the use of iron and steel parts unless they can be adequately protected against the action of water and air.

For connecting the mercury reservoir with the manometer and burette, it is advisable to use a rubber tubing that will not foul the mercury. Such tubing is obtainable under the name of "nitrometer tubing."

Whenever changing the pressure to which the tubing and trap are subjected, care must be exercised to do it slowly, so as not to subject the glass portion of the trap to sudden pressure changes.

The object of the glass portion of the trap is to enable one to see that the surface of the trap fluid is always at the same height whenever the pressure is determined. Exact readings or settings are not required, as a variation of one-twentieth of an inch either way from the line have only a very slight effect on the results. It is therefore possible that in place of glass a tougher translucent material might be used (for example, so-called clear bakelite tubing). This would greatly reduce the chance of breakage and trouble with the trap.

When charging cylinder $A$ with a sample of gas it is advisable to pass the gas first through some form of dust trap. This will prevent dust particles from getting into the tubing and clogging the needle valves. However, the gas should not be passed through a dryer unless a sample of dried gas is desired for some special reason.

Washington, November 21, 1929. 